\title{
Erratum
}

The Visual Computer (1988) 4:40 48

\section{The Mona Lisa identification: evidence from a computer analysis}

\author{
Lillian Feldman Schwartz \\ AT \& T Bell Laboratories, 600 Mountain Avenue, Murray Hill, NJ 07974, USA
}

Due to an unfortunate error, the author's name was mis-spelled. It should read: Lillian Feldman Schwartz 